\title{
The Simulated Group Response Paradigm: A new approach to the study of opinion change in Delphi and other structured-group techniques
}

\author{
Fergus Bolgera, Gene Rowe, Ian Belton, Megan M. Crawford, Iain Hamlin, Aileen Sissons, Courtney \\ Taylor Browne Lūca, Alexandrina Vasilichi, George Wright \\ Department of Strategy and Organization, Strathclyde Business School, University of Strathclyde, \\ 199 Cathedral Street, Glasgow G4 0QU, UK
}

\begin{abstract}
Groups provide several benefits over individuals for judgment and decision making, but they suffer from problems too. Structured-group techniques, like Delphi, use strictly controlled information exchange between individuals to retain positive aspects of group interaction, while ameliorating negative. These methods regularly use 'nominal' groups that interact in a remote, distributed, and often anonymous manner, thus lending themselves to internet applications, with a consequent recent increase in popularity. However, evidence for the utility of the techniques is scant, major reasons for which being difficulties maintaining experimental control and logistical problems in recruiting sufficient empirical 'groups' to produce statistically meaningful results. As a solution, we present the Simulated Group Response Paradigm, where individual responses are first elicited in a pre-study - or created by the experimenter - then subsequently fed back to highly-controlled simulated groups. This paradigm facilitates investigation of factors leading to virtuous opinion change in groups, and subsequent development of structured-group techniques.
\end{abstract}

Keywords: Group judgment; nominal group techniques; Delphi; judgmental forecasting; crowdsourcing; prediction markets; judge-advisor systems; expert knowledge elicitation.

\section{Highlights:}

Structured-group research is hindered by lack of experimenter control and low power We present the Simulated Group Response Paradigm (SGRP) as a solution Simulated groups are created using pre-existing authentic or fabricated responses Simulated groups are highly controlled and open to many experimental manipulations The paradigm allows us to focus on questions about what makes structured groups work

aCorresponding author: e-mail fbolger42@gmail.com

Author note: The work reported here is part of a large project with many personnel. All authors listed here are part of a team who contributed to the larger project in some way or another e.g. developing software and training, constructing experimental materials, running participants, analysing data etc. It was agreed at the outset that all team members should be included on all outputs regardless of their specific contribution. The first two authors were principally responsible for the writing of this paper, the remaining authors have commented on drafts, and are listed in alphabetical order. 


\section{Introduction}

On a variety of judgment tasks, groups have been shown to be more effective than individuals (e.g. Hackman \& Katz, 2010; Hastie \& Kameda, 2005). It is presumed that much of this gain (e.g. in judgment or problemsolving accuracy) comes about from the interaction of individuals, during which novel information may be exchanged, the quality of the individual members' information may be assessed, and new links, ideas, solutions (etc.) may be synergistically developed. There is also some evidence that individuals within groups may be motivated to work harder than alone (e.g. Weber \& Hertel, 2007) - a process generally known as social facilitation (Markus, 1978; Zajonc, 1965).

The advantage gained by taking part in a group is often termed process gain; however, groups may also suffer from processes that can undermine their effectiveness, leading to process loss (Steiner, 1972). It is important to recognise that individuals within groups not only differ in their knowledge and ability to understand and combine information, but also in terms of their personalities and motivations - and groups may be swayed by those who are the most dominant and dogmatic rather than most knowledgeable, or by those playing personal or political 'games' for their own gain. Individuals in groups may also fail to share their unique information, focusing instead on the information that is held in common (e.g. Stasser \& Vaughan, 2013). Further, people in groups may suffer social inhibition instead of facilitation due, for example, to anxieties related to interacting with strangers (Buck, Losow, Murphy \& Costanzo, 1992), reducing their motivation to perform. Social facilitation appears to turn into inhibition as task complexity increases, perhaps due to increasing levels of arousal (Zajonc, 1980) or evaluation apprehension (e.g. Blascovich, Mendes, Hunter \& Salomon, 1999). There can also be a diffusion of responsibility in groups, leading to reduced participation, sometimes referred to as 'social loafing', with the probability of such behaviour increasing with group size and degree of physical and temporal dispersion of group members (Chidambaram \& Tung, 2005). Finally, a number of well-documented cognitive biases - that can reduce the quality of individual judgment and reasoning (e.g. Arnott, 2006; Kahneman, Slovic \& Tversky, 1982) - may be exacerbated by group processes. For example, while individuals are often overconfident (e.g. Johnson \& Fowler, 2011; Lichtenstein, Fischhoff \& Phillips, 1982), groups can lead to even greater risk taking and judgmental confidence than that exhibited by their component members (Dodoiu, Leenders \& van Dijk, 2016; Stoner, 1968). 


\section{Structured Group Techniques}

A number of methods have been developed over the years to harness the positives of groups while pre-empting or ameliorating the negatives, in the hope of increasing the likelihood of process gain. We refer to these methods as 'structured-group techniques' because they use protocols to structure the communications between group members, rather than letting them freely interact. One of the earliest and most widely used of these structured-group techniques is the Delphi method (e.g. Linstone \& Turoff, 1975). An important characteristic of Delphi is that group members - referred to as 'panellists' - never actually meet, thus Delphi panels are 'groups' in name only (hence Delphi is sometimes referred to as a 'nominal group technique'). Indeed, not only do Delphi panellists not meet, they do not even know who their fellow panellists are - a deliberate ploy designed to ameliorate cues related to supposed seniority, experience, or expertise, that may prove to be invalid (as perceived expertise can often be related to personality or social characteristics rather than pure skill or knowledge, see e.g., Bolger, 2018). For example, Burgman et al. (2011) found that 'esteem' indicators - such as years of experience, number of publications, and extent of professional or academic qualifications - are poorly related to performance, while other studies show similar weak correlations between experts' professed confidence and measured ability (e.g. Gibbons, Sniezek \& Dalal, 2003; Phillips, 1999;

Rowe \& Wright, 1996; Rowe, Wright \& McColl, 2005). However, there is some evidence that peer judgments of expertise based on knowledge of the personality of the target can reliably predict forecasting skill (Alvarado-Valencia et al., 2017), and so expertise cues are not always invalid. In any case, anonymity allows nominal group members to focus on the informational content provided by others free from any potentially biasing distractions.

Anonymity is but one of four defining characteristics of a Delphi process: the other three are iteration, feedback and the aggregation (or collation, if responses are qualitative in nature) of group responses (e.g. Rowe, Wright \& Bolger, 1991). Thus, in the typical application of the technique, anonymous panellists are asked to provide a judgment (or forecast, choice, solution, etc.) on the issue at hand in a first round. These individual judgments are then collated by a monitor/facilitator, aggregated, and in some way fed back to the panellists on a second round. Panellists then consider the information (which may simply be the mean or median of the nominal group response, where quantitative values are called for, but may also include rationales or justifications for answers), then provide another response. A number of further iterations may 
take place, continuing until stability of responding is achieved, though most change takes place in the second round, and there may be little point going much beyond this as the advantage of additional rounds must be weighed against the costs of increasing panellist drop out, fatigue, and reduced willingness to participation in future surveys. After the final round, the facilitator aggregates/collates the responses of the individual panellists, and that answer - which is usually equally weighted - is taken as the group response.

The main ideas behind the approach are that anonymity reduces the influence that powerful or dogmatic individuals can have on group judgments; iteration with feedback allows participants the chance to reconsider and improve their responses in the light of information from other group members; and statistical aggregation with equal weighting ensures that the final response fairly reflects the views of all of the group members, not just a few. Further, since there is no face-to-face interaction, groups can be assembled without the need for panellists to be present at a particular location, or to make their contributions at the same time practical benefits when experts are dispersed, perhaps internationally, with limited time and conflicting or busy diaries. The ability to apply the techniques remotely lends itself well to their application on the internet, which may be partly responsible for their growing popularity amongst organizations in both the public and private sectors (e.g., Efrati, 2019; Haynes \& Shelton, 2018).

To be more specific, anonymity, iteration (i.e. rounds of revision), feedback and aggregation achieve the goal of reducing process loss by enabling Delphi to realize the following four principles:

i. The Honesty Principle: All panellists can express their honest opinion (i.e. there are no explicit or implicit influences on opinions such as pre-expressed opinions by other panellists);

ii. The Quality Principle: Each panellist is permitted to review the opinions of other group members in such a manner that they are only influenced by the quality of the reasoning free of potentially biasing indicators of identity, status, confidence, preponderance, order effects etc.;

iii. The Revision Principle: All panellists have the option to revise their original, individual opinion in the light of the feedback received (and while there should be no coercion to change opinion, this might be encouraged, so as to overcome inertia biases);

iv. The Equality Principle: The revised opinion is given equal weight in any further rounds of feedback and the final answer, ensuring that all have a say in the final answer and not just the most voluble. 
Since Delphi was first conceived in the 1950s, the opportunities and demand for judgment and decision making by geographically dispersed nominal groups of individuals has increased dramatically by dint of the emergence of the internet and globalization. Thus, recent years have seen a proliferation of nominal group and 'crowdsourcing' techniques (Brabham, 2008) including, but not limited to, Investigate-DiscussEstimate-Aggregate (IDEA) (e.g. Hanea et al., 2016), Real-Time Delphi (RTD) (Gordon \& Pease, 2006), Judge-Advisor Systems (JAS) (Sniezek \& Buckley, 1995), and prediction markets (Surowiecki, 2005), in addition to the earlier Nominal Group Technique (NGT) (Delbecq \& Van de Ven, 1971).

Before considering factors that affect opinion change in structured-group techniques (and, for that matter, 'real' interacting groups), it is useful to know what the differences are between Delphi and these other techniques, since a sub-goal of this paper is to set a research agenda for understanding the implications for the quality of judgment of changing particular features of structured-group protocols. To make this comparison we will examine each of the structured-group techniques listed above in terms of the extent they permit conformity to the four Delphi principles: (i) true expression of opinion; (ii) judgments influenced solely by quality of reasoning; (iii) opportunity to revise opinions; (iv) equal weighting of each panellists' opinion in any summary or aggregation.

Any technique that involves direct interaction between participants (as opposed to through a facilitator, as in Delphi) is likely to violate one or more of these principles. IDEA and NGT both require individual opinion statements at the outset, and thus comply with principle (i) so long as anonymity is maintained for this step (which is usually the case), but then allow direct interaction between group members, which will usually violate principles (ii) and (iv) of everyone contributing equally in a non-biasing manner, even if anonymity can be maintained (which it usually is not). Both techniques also allow final and private revision of opinion, consistent with principle (iii), but IDEA gives each opinion a different weight in the final conclusion (depending on measures of each member's performance on a related task, the reliability and validity of which are yet to be adequately demonstrated, see Bolger \& Rowe, 2015 a, b), thereby further violating principle (iv).

RTD allows panellists to express their opinions publicly whenever they like within a given time window, thereby violating principle (i), since only the first person to enter will be naïve to any other panellist's opinion. Further, panellists can express their opinions as often as they like, with no facilitator 
control over form or content, thus, as with other direct interactions, they will likely violate principles (ii) and (iv) as not everyone's views will be fed back, since there is no requirement for everyone to respond before continuing to the next round (as there are no rounds). Also, since individual opinions are not collected, vetted and summarised by the facilitator before being fed back to panellists, those views that are fed back could well be biasing in the same way as in freely interacting groups (e.g. dominating by repetition, appeals to authority etc.). Finally, although there is aggregation, since aggregation is in real time it will only include those who have contributed up to that time, so it is unlikely to give everyone's opinion equal representation, and not everyone will get the opportunity to revise their opinion, thus the technique will often violate principles (iii) and (iv). In other words, there is no 'sync point' in which everyone's opinion is based on the same information expressed just once, as in the case in traditional Delphi. In sum, RTD violates Delphi's democratic principles by giving too much weight to panellists who enter early and/or repeatedly.

Precisely how a prediction market is implemented can vary substantially, but the general principle is that each individual participant buys a prediction of an event at a price that reflects the current collective thinking of the probability of that event occurring. For example, if there are two possible outcomes ' $\mathrm{A}$ ' and ' $\mathrm{B}$ ', and outcome 'A' is seen by the majority as being the more likely of the two, then outcome 'A' will command a higher price than outcome 'B'. When the event - say an election - is resolved, the price of the winning outcome will be an amount corresponding to a probability of 1 (i.e. the maximum possible price) and the losing event(s) a probability of 0 . So if, for instance, you believe that there is a $70 \%$ chance of Candidate A beating Candidate B you should be prepared to pay up to $\$ 0.70$ for the chance to win $\$ 1$ (i.e. the expected value of the bet is $\$ 1 \times p(.7)=70$ cents). Prediction markets usually have many contributors - in fact they need a large number to be effective - and their identities and individual opinions will be unknown, so there is anonymity. However, unlike Delphi there is no requirement for participants to consider then express their opinions privately before looking at the market prices, which are an aggregate of all opinions expressed so far. Prediction markets are therefore more akin to an RTD than a classic Delphi in that when participants join they already can see what all previous entrants have said (in aggregate form). There is also no explicit mechanism for revision although, as in RTD, there is usually nothing to stop a participant entering repeatedly by making multiple trades in the market on the basis of new information (or changes in prices). Prediction markets are therefore basically subject to the same non-Delphic properties as RTD, with the possibility of being led by the opinions of early movers in an information cascade, and this - combined with the very limited information 
exchange possible (the prices) and their competitive rather than co-operative nature 1 - could be behind some notable failures of prediction markets (e.g. Brexit and the 2016 US presidential election) (e.g. see Levingston, 2016; The Economist, 2016; Gelman \& Rothschild, 2016).

In JAS, the organization of the judgment group is different to any of the other methods described above in that it does not comprise a collection of peers but rather, as the name implies, has a single judge who is typically also the decision maker - taking advice from one or more independent advisors. There is no interaction between the advisors nor, usually, is there two-way communication between the judge and the advisors: the judge simply sees the advice of the advisor(s) and decides whether to make use of it or not in their final judgment and decision making.

Since in JAS there is only a single judge, to what extent can it be considered to be a structured-group technique? We argue that it can because JASs are comprised of several people - the judge and the advisors whose interactions are structured such that information normally only flows from the advisors to the judge. JAS is, in fact, rather similar to a 2-round Delphi in that (usually) a judge makes a judgment by him or herself first, then receives advice from the advisors (which is comparable to the feedback received from the rest of the 'group' in Delphi), then the judge can choose to revise his or her initial judgment in the light of the advice in the same way that individual Delphi panellists can revise their opinions after feedback. Thus three of the four Delphi features are present in JAS: anonymity (most JAS studies use fictional advisors, although sometimes characteristics, such as their expertise level, are indicated - but this is also sometimes done in Delphi e.g. panellist confidence fed back as an indicator of expertise); and feedback (the advice given); iteration (the possibility to revise judgments after advice, although there is only ever one opportunity to do so). Only aggregation is missing. However, in the analysis of JAS studies there is generally aggregation over all participants (with equal weighting), but this is primarily to assess the level of advice utilization, with assessment of the degree to which opinion change increases accuracy only a secondary consideration, if at all (i.e. changes in accuracy of opinion will be a function of the correctness of the advice and the degree to which the advice is utilised see, e.g. Bonaccio \& Dalal, 2006). Overall, JAS can be seen as the most structured and

\footnotetext{
1 These properties are in contrast to e.g. traditional Delphi where, if a panellist believes his or her judgment to be correct s/he tries to persuade the other panellists of this with good arguments. In PMs there is neither incentive nor opportunity to persuade others that they have the price wrong - in fact you sometimes stand to gain from not stating your true price e.g. pushing the price up then short selling (easier to do than for a traditional market since you usually know when a PM will be resolved).
} 
controlled of structured-group techniques and, as such, we argue, a crucible well-suited to examination of influences on virtuous opinion change. For this reason, as we will discuss further later, JAS is the basic template for the Simulated Group Response Paradigm that we present in this paper.

Although from this analysis JAS and Delphi appear the most rigorous structured-group techniques, this does not necessarily mean that they are always the best, as what is 'best' will depend on an interaction between characteristics of the experts and the task, as well as the goals of the exercise. For example, complex tasks - where there are just a few highly expert panellists whose opinion is divided between several options might be cumbersome to resolve without direct interaction between group members. In this case, NGT or IDEA might be favoured over Delphi, particularly if the goal is consensus rather than accuracy. Alternatively, where there are few options but many potential 'experts', and we are looking for a simple output from the process, such as a probabilistic forecast, then Prediction Markets might be a good choice for achieving accurate aggregate results. The SGRP, to be described below, facilitates the systematic comparison of the various structured-group methods along dimensions hypothesised as relevant, and thus should permit the matching of methods to particular combinations of task, expert and goal.

\section{Consensus vs. accuracy}

We noted in the previous paragraph that a goal of Delphi could be to obtain consensus around a judgment or opinion, or it could be to improve accuracy of a judgment or opinion, or both. We have informally reviewed the literature and found reports of thousands of applications of Delphi in just the last 20 years - most of these are in health provision, and most have the stated goal of obtaining consensus, for instance, around diagnoses or treatments. This focus on consensus rather than accuracy is reflected in the common characterisation of Delphi as a 'consensus method'. NGT is similarly characterised. However, in its original form, Delphi was as much about attaining accurate judgments as it was about obtaining consensus: it was designed to improve the "reliability" of forecasts by using multiple experts who would then "converge" on the best answer free from biasing influences of face-to-face "confrontation" (Dalkey \& Helmer, 1963). But, since the original use for Delphi was long-term forecasting, the outcomes needed to check whether the method actually improved accuracy were not available, whereas convergence was more easily observed. Although validation is possible, for instance, by using short-term forecasting or 'almanac' tasks, there have been relatively few attempts to examine accuracy improvement in Delphi over the last half century - what few there have been we will turn to 
in the next section. Meanwhile, in many of the more qualitative applications of Delphi, consensus is also easier to assess than accuracy. However, 'easier' does not necessarily mean 'easy' and there has been a lack of consistency in the method and rigour of consensus measurement (von der Gracht, 2012), which has negatively impacted on the conclusions we can draw about the effectiveness of Delphi in this respect.

In the current paper our focus will be on how structured-group techniques can improve accuracy rather than produce consensus. There are several reasons for this focus. First, although we are concentrating on Delphi - due to its age, popularity, and because, as we shall see, it has a theoretical underpinning that others do not - we are interested in structured-group methods more generally and, with the possible exception of NGT, the other techniques we discuss have principally been developed to improve accuracy rather than consensus. Second, there has recently been an interest in applying structured-group techniques to improve forecasting (see, e.g., Bolger \& Wright, 2017 and the rest of the Special Issue) - or a revived interest in the case of Delphi - and to the elicitation of uncertain quantities for use in risk modelling, so-called 'expertknowledge elicitation' (EKE, see e.g., Dias, Morton \& Quigley, 2018). In both these cases the emphasis is again on accuracy2 rather than consensus (although the particular method of aggregation is a hotly debated topic e.g. Bolger \& Rowe, 2015 a, b and commentaries). Structured-techniques have also recently been used for assisting reasoning with evidence for intelligence analysis (e.g., Dhami, Belton \& Careless, 2016) and in these cases, accuracy can be difficult to assess objectively, but is still the primary goal and so - in development at least - the success of the techniques is evaluated against normative gold-standards (e.g. Bayes Theorem or logic), if available, or solution rubrics agreed between domain experts if not. Another argument against consensus as the primary goal of a structured-group technique is that sometimes there may be alternative positions that cannot be resolved, in that there is good evidence and/or arguments supporting each, thus decision and policy makers might wish to consider alternative models or scenarios implied (this is, in fact, the stated main goal of policy Delphi (Turoff, 1970)). Ultimately, although having a single consensus view as the outcome of a structured-group process may be helpful for decision making, and hence desirable, it is not much use if the consensus is around a value that is not actually supportive of good decisions.

\footnotetext{
2 In this contemporary use, the forecasts that are elicited are usually probabilistic, so accuracy is qualified by the realism of the uncertainty attached to the forecasts. The same is true when structured-group techniques are used for the elicitation of uncertain quantities for the parameterization of risk models.
} 


\section{Do structured-group techniques improve accuracy of group judgment (and if so, how)?}

In answering this question we will first focus on Delphi before turning to the other techniques described above. The reason for this focus is that, as we have just argued, Delphi can be regarded as the paradigm case from which other later techniques are less rigorous, or complete, alternatives. Further, as we will discuss shortly, Delphi is underpinned by a theory that accounts for movement towards more accurate judgments.

So what is the evidence that Delphi produces better judgments? As we have already mentioned, although Delphi started out equally concerned with accuracy and consensus its use has, until the last few years, largely migrated towards the latter. The consequence of this orientation is that there have been almost no studies in recent years that attempt to evaluate the method in terms of its ability to produce more accurate judgments or forecasts, either during the course of the method itself (e.g. comparing the aggregate judgments from the first and final rounds), or relative to other structured-group techniques or unstructured (face-to-face) groups. Consequently, the most recent review of the accuracy of Delphi was over 20 years ago (Rowe \& Wright, 1999) where it was found that Delphi has often shown improved performance versus interacting groups or individual panellists' statistically aggregated first round responses - at least for short-term forecasting problems and tasks involving judgments of quantities, where accuracy can easily be assessed3. However, we argue that it is important to recognise that this does not imply that the technique will always lead to benefits in accuracy in every situation. Furthermore, our question is made difficult to answer because several different versions of 'Delphi' have been applied (obtained by relaxing or deleting one or more of the four characteristics discussed above). We therefore argue that a more appropriate question to ask is: 'under what conditions is a Delphi process likely to improve accuracy?' This question implicitly accepts that Delphi is neither a single thing nor a panacea to all ills, and invokes a need to answer a series of more nuanced questions about what, how, when, and why. The answers to these questions should help to determine where principles can be relaxed, and where they should be tightened, in order to balance Delphi performance against its practicality.

Of course, accuracy is only one of several possible evaluative criteria. Hasson and Keeny (2011)

\footnotetext{
3 We have scoured the literature since Rowe and Wright's (1999) review and found only two studies that attempted to empirically evaluate the accuracy of the Delphi, both again in the forecasting domain. Parenté et al. (2005) found significant improvements in forecasts between first and second rounds, but not between subsequent rounds, while Graefe and Armstrong (2011) found no overall statistically significant differences in forecasting accuracy between Delphi, NGT, PMs and face-to-face groups.
} 
consider studies up to that date that examined various aspects of reliability, validity and trustworthiness of the Delphi method, of which accuracy forms only a small part. However, Hasson and Keeny have difficulty coming to any firm conclusions for similar reasons to those we discuss here for accuracy (and consensus): the huge variety of uses and forms of Delphi; poor experimental control; small samples; measurement issues; and a paucity of research full stop. We contend that the research paradigm proposed here is equally applicable to answering questions about the effectiveness of structured-group techniques in terms of evaluation criteria other than accuracy (and anyway, accuracy subsumes other criteria in that, for example, consistently accurate - or well-calibrated - outcomes are also likely to be both reliable and credible).

So how might accuracy improve in a Delphi? We propose that the answer to this is through what we call virtuous opinion change (VOC) - that is a shift over rounds of opinions towards the 'truth' - by the individual members of the group, with those individuals who are initially further from the truth, shifting opinion more than those individuals initially closer to the truth. We say 'individual' members, because the Delphi group output is exactly equal to the sum of its individual parts, established through the equal weighting of the responses of all of the panellists. Of course, there is an argument to be made as to whether an equal weighting approach is the best one, and whether this might not be improved upon by differentially weighting panellists' responses, but there is presently little compelling evidence of better schemes than equal weighting (see, e.g., Bolger \& Rowe, 2015a, 2015b), and note that differential weighting would not undermine the principle that the 'group' output of Delphi - and other nominal groups - is an aggregate of the output of individuals. In other words, classical Delphi is unlike directly interacting groups, where some mysterious process might lead to a group output bearing little clear relationship to individual judgments (and might even lead to a final output falling outside the initial range of judgments as a consequence of some synergistic process)4. From this perspective, we argue that the unit of analysis in Delphi studies should primarily be at the level of the individual panellist rather than at the level of the group, the quality of which is almost completely dependent on the individual processes from which it is composed5. Thus, if we understand what individual panellists are doing, and why they are changing their responses (whether in a virtuous manner or otherwise), then we understand the working of the method, and we can make prescriptions as to how to design methods so

\footnotetext{
4 So when we allow direct interaction, as in some other techniques, we lose insight into the sources of VOC. 5 'Almost' because the quality of the outcome could be influenced by post-group processing such as editing by a facilitator or, indeed, performance-weighted aggregation.
} 
as to achieve optimal results in the future.

So how is Delphi presumed to facilitate VOC in groups? In the Theory of Errors (Dalkey, 1975; Parenté \& Anderson-Parenté, 1987) it is suggested that there are two kinds of people in groups: 'holdouts' who are sure of their opinion on the issue at hand, and so do not change over subsequent rounds (or do not change much); and 'swingers' who are not so sure of their position and are inclined to move towards the positions expressed by those who seem more confident and/or expert6 (the holdouts). Thus, over iterations, judgments by a panel will tend to coalesce around those of the holdouts. If there is little disagreement between experts (i.e. essentially a single holdout position) and the holdouts' confidence in their own position is justified (i.e. they actually do have greater expertise than the swingers), then the majority of opinion change will be virtuous, and the end result will be a group judgment that has a higher degree of consensus than initially, and is centred around the 'truth'. In a sense, then, experts weight themselves in the aggregation, so that those who (feel they) have more insight into the truth end up having a greater influence on the final group judgment than those who (feel they) have less insight on the truth.

Of course, there are several threats to the happy outcomes of greater consensus and proximity to the truth being realized. First, confidence may not be well correlated with the closeness of expert judgment to the truth - Bolger and Wright (2011) argue, and provide evidence, that this is sadly the case in some task domains. If the expert-truth correlation is weak then the holdouts may not necessarily be more knowledgeable than the swingers, so we may end up with little VOC, or even average opinion change away from the truth, as meek experts move towards bold non-experts. Another possibility is that there is a high degree of homogeneity in the group so that everyone expresses approximately the same judgment (and thus there is no reason for anyone to change their opinion), which is fine if everyone is fairly accurate, but, of course, not useful if they are not. In either case the Delphi process will be unlikely to produce VOC. On the other hand, if everyone makes different judgments, but are homogenous with regard to how strongly they hold the beliefs underpinning these judgments, then Delphi should produce greater consensus (assuming that the degree of change towards the group average is monotonically and negatively related to the strength with which an opinion is held), although not necessarily a more accurate group outcome (i.e. if truth lies in the margins of the

${ }_{6}$ Sometimes confidence in judgment is fed back, sometimes rationales - which should convey expertise more directly than confidence - and sometimes both confidence and rationales are given. 
distribution of opinions, then move to consensus over rounds will be away from the truth). Yet another possibility is that there are two or more positions firmly held that split the swingers, and thus no consensus is reached, and accuracy inevitably suffers.7

To obtain better outcomes across a range of tasks and panellist attributes it has been proposed that, rather than letting confidence be the sole driver of the process, we should encourage people to provide rationales for their judgments (e.g. Rowe, et al., 1991). This way it is hypothesized that people shift their opinion towards better arguments rather than confidence, the former being better correlated with truth than the latter, however, this hypothesis as yet lacks firm empirical support (for reasons we will shortly discuss). Elicitation of rationales might help in the situation where there is high homogeneity but low accuracy, in that the resulting poor-quality rationales may lead to panellists re-thinking the problem and producing a better solution at a subsequent round. Where there is high heterogeneity of opinion, but homogeneity regarding commitment to these opinions, rationales might help to persuade people to switch to better-argued positions rather than sticking to their original position or being drawn to some arbitrary average. Rationales may also prevent coalescence around alternative positions if a single alternative stands out as better supported.

The Theory of Errors was developed to account for opinion change in Delphi - to what extent can it be applied to the other SGTs under consideration here? In IDEA, NGT and JAS the usual protocol is similar to Delphi in that participants are explicitly required to consider and state their opinion independently first, thereby giving them a position that they can subsequently defend and either hold or switch from as in ToE. However, IDEA and NGT have direct interaction after the first round, providing potential for a range of behaviours - such as publicly forming alliances or flip-flopping between positions - that are not possible within a single Delphi round and are perhaps not well characterised by ToE. In general, the number and type of influences on any opinion change are likely to be greater when interaction is relatively uncontrolled. Meanwhile, it is possible that RTD could either function like a classic Delphi, or it could function more like an unstructured group, without even the requirement to take an initial, independent position: as previously discussed, after the first entrant subsequent entrants could simply copy the position of the first8.

7 As an alternative to use as a consensus method Delphi can be usefully deployed to identify different positions, alternatives etc. that can influence policy making (aka 'Policy Delphi').

8 The degree to which this occurs in reality is an empirical question but probably depends on the expertise and motivation of the participants with respect to the problem at hand, and the infrastructure for polling opinion e.g. it could be made 
While in RTDs an initial independent opinion is at least normally requested - even if it is not given there is usually no such requirement in PMs, so potential participants can simply see what the consensus is (as given by the price) before deciding whether or not to enter. Clearly the ToE is not a good account for the situation where there is no preferred initial position to uphold or abandon, but again the extent to which this situation is common in reality is questionable: it may be the exception rather than the norm that people enter a market that they care so little about that they have no prior opinion of the outcome, and in those case where it occurs, the prior could be set to the price on entry with a relatively low resistance to change on the basis of subsequent market movements. Note that since PMs are generally set up to elicit probabilistic forecasts an alternative to the ToE might be Bayesian updating in the light of information received about price (or departure from Bayesian updating, such as ‘conservatism' (Edwards, Lindman \& Phillips, 1965). A similar approach has been taken in JAS research where the principal dependent variable is 'weight of advice' defined as the degree of opinion change as a function of the difference between the initial judgment and the advice (e.g., Harvey \& Fischer, 1997; see also Sniezek, Schrah \& Dalal, 2004). Where probabilistic judgments are made in structured groups (as in recent work on EKE for risk assessment e.g. EFSA, 2014) a similar Bayesian approach could be taken (or perhaps Brunswikian if there are multiple expert opinions9).

This discussion indicates a number of factors that may influence whether a Delphi-like approach leads to VOC or not, not least: knowledge (perceived vs. real expertise); the degree of variation in that knowledge between experts, and the strength with which it is held; the nature of feedback passed between panellists; the relative balance of this feedback (e.g. minority vs. majority positions); and the extent to which fed-back opinion agrees with each panellists' initial viewpoint. Further, the personal characteristics of panellists (confidence, intelligence, stubbornness, etc.) are likely to influence opinion change, as may the precise nature of the Delphi-like method used (e.g. type and frequency of feedback). Also it is possible (indeed, probable) that the nature of the task being performed (such as its difficulty) impacts on how panellists respond within a Delphi environment (e.g. Hackman \& Morris, 1975; McGrath, 1984; Straus, 1999). All of these possibilities need to be tested as well as numerous other possible strategies for improving the outcomes of structured-group

difficult to see others' opinions before making one's own (but then this could be 'gamed' by entering some nonsense simply to gain access) 9 A potential difference between JAS and PMs - as often implemented and studied - and Delphi i.e. in the latter, variation in 'advice' is an important driver of VOC in ToE, but in the former this information is not available as there is often only one advisor (JAS) or only the mean of 'advice' is given (PMs). If there are multiple sources of advice in JAS then advice utilization is measured by multiple regression (cf. cue utilization in the Lens Model (Brunswik, 1955)). 
processes.

Returning to our initial assertion that structured-group research should refocus on the conditions in which it is likely to improve judgment accuracy, a more precise framing of our research agenda is:

Structured-group research should seek to understand how virtuous opinion change is achieved in individual panellists as a consequence of the interaction of three broad factors, namely, the characteristics of the individual group members; the characteristics of the technique itself (i.e. the particular variation used); and the characteristics of the context in which the technique is used.

There are, of course, many different factors within the three dimensions mentioned here. In Figure 1 we indicate several possible influencing factors within these categories that might be the objects of research in the future (or may have been in the past). The factors indicated in Figure 1 are not comprehensive, but rather illustrative, reflecting an initial proposal of research topics (we invite others to expand or amend our suggestions). The figure also implies a clear separation between the three dimensions, when of course we might expect interactions between the factors within these too, for example, problem type may affect nature of feedback and its potential length and complexity (etc.).

$<$ Figure 1 about here>

\section{Studying Virtuous Opinion Change in Structured Groups}

What does the above framework say about how we should study Delphi and related methods? Before trying to answer this question we should ask how structured-group techniques have predominantly been studied in the past, and just as pertinently, not studied? With regard to Delphi, we have already noted the paucity of empirical investigations of the technique compared to the vast majority of publications that simply report implementations and case studies. The same is true for NGT, while PMs, RTD and IDEA are relatively new, and have not yet generated more than a handful of relevant empirical evaluation studies between them. There are not a huge number of studies of JAS either, with research slowing in the years after Bonaccio and Dalal's (2006) review. However, the nature of the empirical research into JAS is rather different to that into other structured-group techniques in that, rather than simply evaluating whether the methods work in terms of improving accuracy - either in an absolute sense, or relative to other techniques - it has tended to investigate the conditions under which the method works (see e.g. Bonaccio \& Dalal, 2006), or equally, does not work 
since early JAS studies found a reluctance amongst judges to take advice on board (which has come to be known as 'egocentric discounting', see e.g., Yaniv \& Kleinberger, 2000).10

We argue here that rigorous research into Delphi and other structured-group techniques, with the exception of JAS, has been held back, not just by focussing on the question of 'does it work?' as opposed to 'how does it work?' as we argued above, but also by its dependence on running and analysing real groups. By 'real groups' we mean individuals participating in groups constituted for a particular amount of elapsed time, in which they receive feedback from other panellists from their group on rounds subsequent to the first. For example, to achieve significance in the study of Delphi using such real groups, and in which the group is the level of analysis, a very large number of panellists would typically be required for the analysis to have sufficient statistical power to stand a fair chance of observing an effect even if it is of a moderate-to-large size. By contrast, JAS research has not been so constrained, as much of it has either used just one advisor (see e.g., Bonaccio \& Dalal, 2006), and/or fictional advisors (e.g. Price \& Stone, 2004; Sah, Moore \& MacCoun, 2013) and this might account for the generally more methodical nature of the research into this method we observed above.

Let us consider the situation when individuals are the level of analysis. In order to attain a conventionally acceptable level of power (0.8) when using a t-test for two independent groups with an alpha of .05 and an anticipated moderate-to-large effect size (.5) one would require more than 60 participants per condition (and more than twice as many for a more-usually encountered, but still consequential effect size of .35). When we move from individuals to groups we expect to gain some advantage by the reduced variance of the group outputs (due to increased consensus and effects of aggregation), but we still end up having to run nearly as many groups as individuals, say 50-100 groups per experimental condition rather than 60-120 for the set-up described above (note that the power calculations cannot be properly done in advance of measurement of the degree of dependency between group members11). Naturally, each group will comprise several members, and so our figure of 50-100 would need to be multiplied by the group size to establish the needed number of participants - which typically translates into a rather large number of experimental participants (i.e.

10 To be clear, in addition to this JAS work there are several extant studies relevant to the question posed here of the conditions under which structured-group techniques produce VOC. A review is outside the scope of the current paperwhich is to present a new paradigm to accelerate research - but there is no question that there is very much still to learn. 11 This dependency will vary according to both characteristics of the task, such as degree of uncertainty and number of alternative solutions, and those of the panellists, for instance, the heterogeneity of their experience. 
250-500 per condition for groups of five). Logistically, running such a large number of participants is problematic in several respects.

If one wishes to make use of panellists who have some degree of expertise on the topic of study12, as opposed to, for example, university students with no specific relevant expertise (as is standard in empirical academic social science studies), then it can be hard enough to recruit tens of people never mind hundreds. Added to this, in the real-world, drop out of participants between rounds is common and can lead to further difficulties in analysis, as groups initially constituted to have the same size mutate over the process to subsets of different sizes (see Bolger, 2018, for a discussion of expert recruitment and retention). Furthermore, if one were to compare Delphi groups for a single experimental condition to freely interacting groups as the control (as this is a typical benchmark for group performance), then one would need to double the number of participants one needed to recruit - and adding additional experimental conditions with real groups exacerbates the problem still further. In short, conducting research using real groups and doing analysis at the group level is a logistical nightmare, which, even if conducted successfully, is only likely to yield findings after much expense and effort - on one or two variables or variable combinations (and for the inadequacy of this, see the multiplicative complexity implied by Figure 1).

In view of the considerations discussed above, if we wish to learn about the drivers of virtuous opinion change in Delphi, and other structured groups, we need a radically different approach to that of employing 'real' experimental groups and then analysing results at the group level.

\section{Past Studies using Simulated Responses}

The use of simulated groups has a long history in social psychology, arguably beginning with the work of Asch (1951), who used groups of actors (or 'confederates') to provide pre-arranged responses in order to observe the effects of conformity on unaware experimental subjects. However, research on the process of replacing real participants with confederates in nominal groups such as Delphi - which would seem less problematic than for face-to-face groups, as it does not rely upon the acting qualities of confederates, or the need for them to control non-verbal behaviour - is surprisingly difficult to find. In fact, when real participants have been replaced in such research, the dominant approach appears to involve replacing all participants,

12 PMs are usually different from the other structured-group techniques considered here in that they typically use larger numbers of less expert participants. 
notably by running group simulations on computers instead. For example, Meijering, Kampen and Tobi (2013) used computer simulations to create different Delphi scenarios in order to compare the consistency of different 'indices of agreement'. Similarly, Birko, Dove and Ozdemir (2015) tested consensus indices by simulating a three-round Delphi survey in which they varied the number of survey questions, the sample size, and the extent to which experts conform to group opinion. Importantly, neither of these studies used the input of any real participants, and so their results do not account for true human behaviour or provide answers to actual judgment, forecasting or problem-solving tasks (although this is the primary rationale for running a Delphi). Certainly, we are aware of no systematic, well-defined approach to manipulating nominal groups in order to exert greater experimental control in research - which we believe to be a significant omission.

\section{The Simulated Group Response Paradigm}

To aid in the conduct of research in this area, we suggest that research should focus on identifying how to achieve VOC in individual 'group' members, and that this is best done using simulated groups in which the nature and quantity of feedback from supposed fellow panellists (who may or may not be real) is manipulated in a controlled manner in order to allow the answering of precise experimental questions with sufficient power to enable the obtaining of statistically significant results. We call the protocol we have developed the Simulated Group Response Paradigm (SGRP). We use the term 'paradigm' deliberately, as the approach which is depicted in Figure 2 and elaborated subsequently - involves a general methodology within which are a variety of specific procedures that are informed by our theoretical outlook, which is that the intent of research should be to establish and explain the factors (and their interactions) that are responsible for VOC in individuals 13 .

The SGRP consists of two main phases, which we briefly describe now in reverse order.

The second phase is where hypotheses about influences on virtuous opinion change are tested in a Delphi procedure with - in the basic form - two rounds. In Round 1 participants 14 individually provide answers to a judgment or reasoning task. At Round 2 the participants then receive a number of other answers to the same task and are invited to revise their Round 1 answer. Thus Phase 2 of the SGRP is, from the point

\footnotetext{
13 We are thus using 'paradigm' in Kuhn's (1962) specific sense of a theoretical or conceptual framework that guides hypothesis generation, and construction of experiments to test these hypotheses, rather than a more general sense of an example or worldview. We will return to this point in the Q\&A.

14 We use this term instead of 'panellists' as those involved may never become a member of a Delphi-like group.
} 
of view of a participant, identical to a standard two-round Delphi. There are, however, important differences from the perspective of the experimenter, both preceding Phase 2, and after Round 2 of Phase 2 in the analysis of the data. The most significant difference is that the answers fed-back at Round 2 do not derive from other group members working on the task more-or-less synchronously - as is usually the case - but are elicited, or created, at some earlier point in time during SGRP's Phase 1. Thus at Phase 2 there are no 'real' groups interacting through a facilitator, and the unit of analysis is the change in each individual participant's answers between the two rounds.

$<$ Figure 2 about here>

We now consider the two phases in more detail.

\section{Phase 1: Stimulus Creation/Collection}

There are three basic options at Phase 1: create stimulus items to be used in Phase 2 de novo; adapt some existing stimuli to suit your purpose; or elicit responses that can be used in Phase 2 during a pilot study that we refer to as a 'stimulus elicitation exercise' or SEE.

In some circumstances it might be clear what possible responses there could (sensibly) be from participants in a Delphi. For example, perhaps the task of interest is judging the value of an uncertain quantity (but with a known or soon-to-be-known value) that can only fall within a certain range, and your research questions concern the potential biasing effects of different distributions of answers around the true value (e.g. generally too high or too low). Similarly, more qualitative responses might realistically fall only in certain categories, or types, which could be organized in different ways to systematically investigate the relative contributions of the draw of credibility or of a majority. A further possibility could be to generate responses according to some theoretical model of experts, tasks or some combination of the two. For instance, potential distributions of judgments of uncertain quantities might be generated on the basis of assumption that the experts are Bayesian, or deviate from Bayes in plausible ways, for example, 'conservative' updaters (e.g. Edwards, Lindman \& Phillips, 1965) for tasks that vary in terms of the quality of the feedback available. While this approach is perhaps applicable only for generating quantitative responses, there will still be many applications, for example, in forecasting and risk assessment, and later we also suggest how it may be used to study and refine PMs. 
In other circumstances there may be an existing set of stimuli from published research that can be used to test a new research question (or perhaps attempt full or partial replication of an existing finding). Another possibility is to use responses already collected in a previous SEE - by yourself or other researchers: indeed, we encourage researchers in this area who use the SGRP to store any stimuli they elicit in a publicly accessible database that can be re-used by interested parties in future studies.

If stimuli cannot credibly be created de novo and cannot be drawn from previous research, then it is necessary to conduct a SEE.

\section{The Stimulus Elicitation Exercise}

This begins with what is essentially a traditional first round Delphi process. In this, participants are instructed (as per a typical Delphi) to answer one or more questions of interest with an expectation that their answers will be used as feedback to a nominal group: it is important that participants have the expectation that their answers will be used in this way so as to motivate them to produce responses that have value to others, and thus keep the process as similar to a Delphi as possible. Typical questions used in Delphi studies are almanac questions (e.g. 'which is further north, London or New York?'), or short-term forecasting questions (e.g. 'which team is likely to win a tournament?'), but there is no reason why researchers need to be restricted to these, as the mechanisms by which Delphi produces VOC may apply to other tasks, such as logical reasoning or evidence evaluation. Meanwhile, participants might range from the totally naïve to those with considerable expertise on the topic of concern, depending on the research questions and the practicalities of recruitment. Under normal circumstances, however, we advise that the SEE participants be drawn from the same population as those who will be used in Phase 2 (again to maintain compatibility with the standard Delphi processi5).

In this initial step - 'Collect responses' in Figure 2 - considerable information should ideally be collected from participants, not just answers to the questions. Such information could include rationales for judgements (pro arguments), rationales for alternatives (asking participants, for example, 'why might your answer be wrong?'), confidence ratings, self-ratings of participants' own expertise on the topic, and so on.

\footnotetext{
15 It is possible to imagine exceptions to this rule, though. For instance, you might wish to run an experiment with a setup more akin to JAS than Delphi, in which case you could use experts in the SEE and naïve participants in Phase 2. See the Q\&A section later for commentary on wider use of the SGRP.
} 
Responses to one or more personality questionnaires might also be collected in order to establish participants' characteristics (see Figure 1)16. Importantly, the SEE is an opportunity to collect as much potentially relevant information as possible, not only on factors that are of immediate interest to the researcher, but also on factors that may be of interest in the future.

In our view, this first step is then best regarded as a broad data acquisition stage and accordingly we propose an optional second step - 'Collate responses' in Figure 2 - which involves creating a database of SEE responses for each question posed. In this, all of the data from the SEE is collated into a single database, with each participant essentially supplying one row, and each column indicating some characteristic of the participants' data, including their answers and rationales, any ratings they give (e.g. expertise, confidence), as well as any scores on personality measures.

In a third potential step - 'Categorize responses' in Figure 2 - one can apply some categorisation or manipulation to the data collected. For example, one analysis might simply involve conducting a word count of the number of words within rationales given by participants - as it is possible that something as simple as argument length might prove an influencing factor on opinion change over rounds (the main criterion measure). However, more sophisticated analyses could also be done, such as getting a set of ratings of arguments for their quality or complexity or persuasiveness. The key is that the collated material in the SEE database is a resource that can be used in a series of studies, and can be re-analysed and rated according to new hypothetically interesting factors that emerge later. Indeed, there is even the possibility that large databases could be made open source, for use by the entire research community.

It should by now be apparent that the SGRP can be used in at least two different ways: in a deductive mode the experimenter will start with one or more hypotheses and develop stimuli to test these, perhaps necessitating a SEE if appropriate stimuli cannot be found elsewhere or constructed de novo; alternatively, in an inductive mode, data can be collected prior to the formation of hypotheses. In the latter case, after categorization there would be a fourth step - 'Construct hypotheses' in Figure 2 - where research questions can be identified and specific hypotheses formulated (of course, in deductive mode you would skip this step since you would already have constructed your hypotheses at the outset). According to our framework described in relation to Figure 1, we suggest that these hypotheses should generally be of the form that 'factor 
$\mathrm{x}^{\prime}$ (relating to one of the columns in the database produced during collation) is in some way related to a criterion measure of (virtuous) opinion change. Hypotheses developed here might not just involve main effects of factors, but also interactions. For example, one hypothesis might be that the complexity of arguments submitted as rationales will only positively influence the probability of $V O C$ when supported by a majority of panellists - suggesting an interaction between argument complexity and level of support.17

In a fifth SEE step - Figure 2, 'Curate responses' - the appropriate stimulus material for the desired Delphi experiment (i.e. quantitative and/or qualitative responses to the task) is selected, and possibly edited, for use as feedback to real participants in the second Delphi round of Phase 2. This curation of responses for feedback is an integral part of a traditional Delphi process where the facilitator takes responses from each individual panellist and gives them as feedback to the group as a whole, usually after processing the responses in some way. For quantitative forecasting this 'processing' typically involves computing some statistics, such as the mean and standard deviation of the forecasts, which are then fed back alongside, or instead of, the original responses. Where qualitative responses are given, including rationales, the facilitator might, for instance, edit the language to assist comprehension or maintain anonymity of the panellist, or combine several similar arguments into one so as to reduce majority/minority effects. Of course, in some instances, the facilitator may do nothing more than simply collect the Round 1 responses together and pass them back to the group.

\section{Stimulus Selection}

In the SGRP, the experimenter minimally performs traditional Delphi facilitator roles and in addition will usually need to select particular stimuli from a larger set for presentation to the participants at Phase 2, Round 2. In other words, unlike a traditional Delphi, there will not just be $n$ responses to be curated for feedback to $n$ panellists (where $n$ is the size of the panel) but from tens to thousands of stimuli from which $n-1$ must be selected (i.e. the group size less the response of the target participant). There are various ways to do this selection, the choice of which should be determined by the purpose of the study being conducted.

\footnotetext{
17 If one has hypotheses at the outset then they may well shape the SEE e.g. if one wanted to manipulate quality of arguments, then one might ask SEE participants to each produce several arguments with the hope that those produced later will be less convincing than the first ones produced. However, it is not essential to construct hypotheses before the SEE e.g. Phase 1 rationales for forecasts could be categorized in some way after the fact - perhaps as different types of causal explanation - then one could formulate a Phase 2 experiment to test hypotheses about the relative influence of these different types of explanation on VOC.
} 
The first approach is Random Selection. If a proposed study is largely exploratory, with no a priori predictions or insights, then the simplest approach is to randomly select the requisite data from the SEE database. For example, if there are 20 entries in a database and it is intended to study nominal Delphi groups of size six, then data (solutions, rationales) from a quarter of the sampled participants (five) would be obtained from the database using a random selection process, and the process repeated for each nominal group created (i.e. the set of responses fed back to a single participant at Phase 2). For example, if there were 50 Phase 2 participants it would be necessary to generate 50 sets of five randomly selected SEE responses to create 50 nominal groups of size five. Of course, one caveat is a need to be wary about the comparability of the originally sampled participants to the experimental panellists: if these come from a different population then the initial responses might seem rather anomalous to the new sample, with uncertain consequences (for example, imagine using lay panellists and then using rationales from expert participants as feedback; the panellists could be concerned and doubt the credibility of the process) 18 . We will return to discuss the composition of SEE and main study samples - their nature and size - in our final Q\&A section but as a general rule, we recommend as far as possible sampling participants from the same population for both phases of the SGRP.

Random selection is also the selection approach that is most akin to using natural groups in a Delphi. In fact, if your SEE selection is representative of the responses produced by your population of interest (which may mean that it is from a large sample, but not necessarily - potential variation in responses could be small for some tasks) then random selection should lead to SGRP producing the same changes in individual opinions as would be found using traditional Delphi panels (but, as already noted, with greater efficiency, since all observations are independent in the SGRP). Random selection is therefore well-suited for running SGRP control groups against which performance of participants in experimental conditions - with manipulated (i.e. non-random) responses - can be compared.

Often we will want to exert more control over the stimuli that are fed back than with random selection. For instance, we may not have a very large SEE database relative to the degree of potential variation in stimuli. Random selection could lead to some kinds of responses being represented infrequently or not at 
all. If we have some understanding of the relative frequency of different kinds of responses in the population, then we can use 'representative' selection (see next paragraph), otherwise we might simply try to balance the types of responses by randomly selecting from within types, otherwise known as 'stratified' selection. For example, we may wish to balance right and wrong responses, long and short, or quantitative and qualitative. If our stimulus set is rather small, then we may just want to use all of the responses and form them into a number of sets that are matched on some criteria. For example, if we have 20 responses, we could form five sets of four with each quartet formed of one long and correct, one long and incorrect, one short and correct, and one short and incorrect answer. With such an approach we can test to see if there are any differences between our five sets - if matching has been successful then there should be no statistically significant differences between the sets. These and other methods for constructing stimulus sets which, while not strictly random, attempt to maintain some degree of randomness we refer to as Pseudo-Random Selection 19.

A third approach is to make a Representative Selection, in which the participants' responses are selected from the SEE database for inclusion as feedback in the prospective study on the basis of the degree to which database participants, or their responses, are representative of their respective populations in the real world, on certain criteria deemed of relevance to the researcher. For example, consider a question in which participants were asked in a SEE to choose between two options (e.g. 'which is further north, Rome or New York?'): if two thirds of the population choose New York and one third Rome, then a representative selection approach would lead to using responses from the database that matched that ratio. So, if the intent in the prospective study was to use groups of nominal size seven, then four of the six simulated responses would be taken from participants who had chosen the more popular option, and two from those who had chosen the less popular one. Representative selection could be based on all sorts of factors, from demographic or personality characteristics of database participants (e.g. half male, half female), to numerical or qualitative aspects of elicited responses (choosing stimuli representative according to word length of rationales, average number of arguments, etc.). Of course, there are difficulties with implementing representative selection, not least in determining what patterns are 'representative of the population in the real world' as this may depend on there

\footnotetext{
19 A tutorial on sampling methods is beyond the scope of this paper: we simply wish to give a sense of the main options available to researchers wishing to select stimuli in the SGRP (and we use the term 'selection' to refer to determining responses to be used, whereas 'sampling' to refer to choice of participants). Please note that we are using 'pseudorandom' in a looser sense than the more formal one referring to number generation by a precise mathematical procedure that yet still satisfies at least one statistical test for randomness.
} 
being a large body of relevant previous research, while justifying relevant criteria for judging representativeness can likewise be problematic.

A fourth approach to selecting feedback from the database is more apt when you have a specific research question in mind - for example, you want to study the effects of particular types of feedback to answer 'what if?' questions - in other words, using the SGRP in deductive mode. We refer to this as Informed Selection: data is chosen from the database informed by the research questions. As a case in point, one issue that has been of concern in social psychology more generally (as well as in Delphi research) is the extent to which people are affected by whether they are in the majority or minority with regards their initial opinion (e.g., Asch, 1951; Moscovici, 1985; Swaab, Phillips \& Schaerer, 2016). If you wished to test the factors that might be likely to make a minority more influential than is usual, then clearly you would need to select from the SEE database an imbalance of responses, so that most argued for one option, judgment or problem solution, rather than another/others. This imbalance might not reflect the actual ratio of participants' responses to the question in the database, and hence would require a specific rationalised choice of answers from the set (and so the chosen answers would likely differ from those that would be selected using the Random or Representative Selection processes). However, even in such a case, an analysis of the SEE database would likely be useful, as this would show the predominant opinions of the wider population, and thus - for example - if you wished to study minority influence in particular, you would have a good idea as to which option would likely be the minority one in the experimental sample of panellists.

As already mentioned, stimuli collected in SEE might be edited before being used as feedback in the experimental Delphi. However, if one is using informed selection this editing might go beyond simply correcting spelling or grammatical errors, or amending language to appear more professional or easier to read, to actually constructing new feedback. This might be done by significantly amending responses (e.g. turning a positive argument into a negative one), or by actively writing novel feedback, perhaps to fill conceptual holes in one's dataset (e.g., an argument that was never made by respondents to the SEE, but which hypothetically might have been), or indeed, introducing somewhat outrageous or outlying arguments if there was a wish to study, for example, the effect on possible VOC of sensational or extreme opinions (e.g. 'fake news'). Importantly, even if one is extensively modifying the stimuli, the SEE exercise still fills an important role by revealing the views of a typical sample and the language and terminology used - and hence acts as a 
benchmark against which the novelty of devised material could be contrasted.

Finally, it may be the case that we do not wish to select responses at all. This is most likely to be the case if we have not performed a SEE and simply have a set or sets of responses that we have constructed for a particular purpose. For example, for a forecasting task with only two possible outcomes we could manipulate the ratio of responses favouring each alternative in order to investigate majority-minority effects.

Alternatively, it is possible that a SEE database might throw up essentially very few different responses (or different types of response) for some tasks and thus every single response (or exemplar of every type of response) might be used as feedback in Phase 2 (i.e. little or no stimulus selection).

\section{Examples of use of stimulus selection alternatives}

An example of where random or representative selection might be used is in a Delphi study looking at the impact of quality of fed-back responses (e.g. persuasiveness) on VOC. Imagine an experimenter who thinks that there could be a relationship between quality and quantity of feedback, but has no clear hypotheses as to the precise nature of this relationship. For example, it might be that high-quality lengthy arguments lead to more VOC because they seem to be indicators of knowledgeable panellists, or it might be that participants are reluctant to read lengthy feedback closely and thus long rationales produce less VOC. In such a case, the experimenter might simply decide to randomly select from all responses rated high quality to form one experimental condition, and randomly select from all responses rated low quality for the other condition (i.e. let length vary randomly across the two conditions). Alternatively, the experimenter might determine the mean and standard deviation of lengths of responses across those in the entire database and select a set of responses for both conditions that vary only by plus or minus one standard deviation from the mean length (i.e. hold length constant in the samples at a value that is representative of the population). In this example, the representative approach tells you the effect of quality on VOC for the length of responses typically given in the task at hand - which might be of interest from a practical standpoint - and should work better for small samples of responses than the random approach, which might be subject to bias in such instances. On the other hand, if there is a strong relationship between quantity and quality it might be difficult to find sufficient numbers of responses for both conditions when using the representative approach.

One reason that experimenters might wish to use Random or Representative Selection is so that they can claim that their findings are somehow similar to those that might be attained, for that particular question 
and sample, had they done a traditional Delphi with actual groups. Such a claim might be important if, for example, the experimenter wanted to make an argument regarding Delphi performance (i.e. going back to the 'does Delphi work?' question, but with an ability to at least deconstruct why the answer was 'yes' or 'no'). However, if used in this way care needs to be taken regarding assumptions about the base data set - that is, before making such conclusions, it would be important to ensure that the original dataset truly included the full and relevant range of responses - we return to consider how to achieve this in the Q\&A section.

Alternatively, an experimenter might have some evidence that a higher quantity of feedback does generally lead to increased VOC, but that there are mediating factors. For instance, the complexity of language used might interact with quantity such that lengthy feedback comprising more multi-syllabic terms, or more expert jargon, leads to more VOC than high quantity feedback that does not have these properties. Further, it might be the case that without the complexity or jargon, high quantity feedback has no advantages compared to low quantity feedback. Such findings would have important implications with regards selecting experts for real-life groups - structured or otherwise - and/or the provisioning of instructions to help experts become more positively influential within a group. In this case, an Informed Selection would seem warranted. Thus the experimenter would consider responses in their SEE, and select arguments from respondents that suit the requirements of their hypotheses: that is, arguments of different length (feedback quantity) and erudition. The importance of the SEE is that the information elicited might include various arguments and their elaborations that the experimenter might not have thought of independent of the exercise. Further, the SEE would provide a template for realistic feedback should the experimenter decide to revise existing material, or develop new material, thus reducing the chance that this new material would be - or seem to be - artificial and unlikely in the perceptions of experimental panellists.

\section{Phase 2: Experimentation}

\section{Main Study}

In this phase, the main experimental20 Delphi study is conducted. We suggest using two rounds, because most response change occurs after the first round and because this is also experimentally easier (although more

20 We use the terms "experimentation" and "experimental" here deliberately to emphasize that although Phase 1 might be either deductive or inductive, we see Phase 2 as being primarily a deductive one i.e. we would expect that researchers normally enter Phase 2 with an hypothesis or hypotheses to test. This is in accordance with our research agenda as set out in the Introduction. 
prolonged and elaborate designs are possible as we will discuss later in the Q\&A section). Usually a new set of participants are recruited for the main study (i.e. those who have not previously taken part in the SEE21): as already mentioned, in order to reproduce a typical Delphi panel of peers, these participants should be sampled from the same population as those from whom responses were collected in the SEE. At Round 1, each participant individually responds to the target problem(s). Note that these first-round responses could be collected and further added to the SEE database thus enriching it: although the feedback for the second round would have already been identified, the stimuli could potentially be used for future experiments. Next, all of the participants in Round 2 receive feedback, specific to their experimental condition, from a set of anonymous, nominal others (ostensibly those in 'their group'), to consider and use as a basis to refine their own initial judgements (or not).

\section{Main Study Analysis}

Finally, the responses from the Phase 2 experimental study are analysed in order to answer the research questions. Importantly, the level of analysis is usually at the individual level, in which the effects of specific, controlled feedback on each of the $n$ participants in each condition can be established: in other words, the amount of opinion change is recorded for every participant, and assessed as to whether it is 'virtuous' or not. As we have already discussed, since we are focussing on individual outputs rather than on group, and thus have independent observations, we only need $n$ participants rather than approximately $n$ times the group size for inferential statistics to be performed with sufficient power to enable the research questions to be answered with an acceptable degree of confidence.

To give a specific example of how SGRP can allow questions about effects on VOC to be answered efficiently let us consider how we might examine the influence of group size on amount and quality of opinion change. Presumably there is usually a trade-off between the advantages of adding knowledge and perspectives by increasing group size, and the disadvantages of increased information load for panellists and facilitators, not to mention the practical difficulties of finding and co-ordinating larger groups of experts. To compare just two group sizes - say 5 and 9 - in a traditional Delphi set-up one would need to run 60 groups per condition (for power .8 , effect size .35$)$ meaning finding $840((60 \times 5)+(60 \times 9))$ participants! This is probably why such a study has never been done, to our knowledge (the two extant studies of the effects of group size in

21 It is possible, although logistically problematic, to reuse SEE participants, as we discuss in the Q\&A. 
Delphi have a number of shortcomings that we will address briefly below in order to make our point regarding the difficulties of conducting such research in the 'traditional manner'). In the SGRP one needs 120 participants (2 groups of 60) plus possibly another group for the SEE - the exact number depending on the task, but is unlikely to be more than a further 60 - to answer the same question (note that increasing nominal group size does not affect the number of participants needed in Phase 2 of SGRP but may impact on the number needed for the SEE in Phase 1); this could still be problematic for some expert-by-task combinations but a huge improvement over the traditional method, nonetheless. An added advantage of SGRP over traditional groups is that, since participants are working individually, they can do so whenever is convenient for them and/or the experimenters rather than having to co-ordinate panellists - in our experience, such coordination can be a substantial drain on researcher resources. Finally, it is worth noting that we would normally want to compare more than just two group sizes and that there will probably not be a universal answer to the question "what is the optimal group size for Delphi?" as it will no doubt depend on each of the three factors in Figure 1: thus answering the question will mean many multi-condition experiments, which we contend would be impossible in practical terms without using the SGRP.

The only two studies to date that have directly examined Delphi group size found no consistent relationship between group size and effectiveness. Boje and Murnighan (1982) compared the performance of Delphi groups of 3,7 and 11 across two statistical and two almanac problems with answers uncertain or unknown to participants respectively. The performance measure was accuracy of group numerical estimates. Estimate accuracy slightly decreased with group size but confidence significantly increased, thereby inflating overconfidence. In our view this finding is most likely due to a lack of expertise in these student participants regarding the problems posed and/or a lack of motivation. However, to use real experts would likely have further exacerbated the already low power of this study - the group-level analysis was based on a maximum of 7 points, the largest number of groups in a condition - thus making it unlikely to discover effects of group size on accuracy even if the effects are relatively strong.

Brockhoff (1975) compared Delphi groups of 5, 7, 9 and 11 panellists. Group performance was measured using the median group error on sets of "fact-finding" and forecasting questions. The study found no systematic effects of group size, with the ordering of groups by accuracy varying as a function of problem type and round assessed (of which there were five). Although the participants in this study were 'experts' 
(familiar with the problem domain - finance - although not especially with the tasks, particularly fact finding, which apparently they generally considered pointless), power was even lower than in the case of Boje and Murnighan (1982), as only one group of each size was tested. In both studies many details of what was actually done are vague - particularly the precise nature of the feedback to the panellists - and we suspect that there was poor experimental control. The value of the findings of these two studies is consequently somewhat limited, and we still have no real idea of how varying group size affects VOC and the resulting accuracy of the Delphi output.

Aside from this greater efficiency, we posit the main advantage of the SGRP over 'real' Delphi groups is in experimental control. What the approach enables us to do is essentially answer 'what if' questions: what if participants had certain qualities, or the feedback was of a certain type, or the process was structured in a certain way, or the questions to be answered were of a certain type? Answering these questions not only allows academic learning about what factors in nominal groups are effective in stimulating virtuous opinion change (or any change), as well as testing theoretically interesting hypotheses as to why these may prevail, but also has practical implications. If we have a 'real world' Delphi problem to solve, with real experts, the appropriate SGRP studies should be able to inform exercise sponsors about the type of experts to recruit, in what numbers, and using which processes, in order to enhance the likelihood of getting improved judgments. Compared to this, traditional Delphi empirical studies with 'real' groups essentially rely upon serendipitous recruitments and interactions to yield convincing and publishable results, and absence of control means that all most studies can say is that 'on this particular occasion, this particular assemblage of participants happened to be aided by this particular variation of the Delphi process'22. As to the question of whether a similar result would be obtained under any minor variation of the factors, such traditional studies would likely be mute.

\section{Discussion}

Throughout our presentation above, a number of questions occurred to us with regards to the use of the SGRP for the development of theory and practice, which we set aside in order not to interrupt the flow of our

\footnotetext{
22 Journals have a preference for research that reveals findings in which one can have some confidence, usually indicated by demonstrating the statistical significance of claimed differences between conditions, with a bias against studies that yield null results (e.g. Rothstein, Sutton \& Borenstein, 2005). We can only speculate on the number of unpublished empirical studies of Delphi languishing on researchers' hard-drives.
} 
argumentation. In the penultimate part of this paper, in place of a discussion, we present these questions and attempt to answer them.

\section{What is the relationship between SGRP and traditional structured-group research?}

We anticipate that many readers will be interested to know the extent to which the SGRP can substitute for traditional structured-group research with 'real' groups. Predictably, perhaps, the answer is "it depends..." While it is possible to imagine variants of SGRP that are to all intent and purposes identical to traditional Delphi - and we will touch upon such designs briefly below in the answers to a couple of the other questions raised - such an approach loses much of the point of the SGRP. This point being - as discussed above in relation to the paradigmatic nature of SGRP - to assist the design of tightly-controlled experiments for answering questions about influences on opinion change and, as such, the principal raison d'etre of SGRP is not to simulate real groups but rather to remove aspects of real groups that prevent the efficient investigation of some important research questions: using SGRP to simulate Delphi with real groups inevitably means reintroducing that inefficiency and poor control. We made a similar point earlier with regard to the consequences of allowing direct interaction between participants in a structured group, thus this is clearly an even greater issue for those techniques which do - NGT and IDEA - but less of one for those methods where interaction is more constrained than Delphi: PMs and JAS.

Having said all this, as we will illustrate shortly, there may still be circumstances when one would wish to use more complex or 'realistic' SGRP designs. Meanwhile, considering a basic SGRP with just two rounds in Phase 2, as presented in Figure 2, although from the viewpoint of a participant the experience is the same, there is an important difference between the output of an SGRP 'group' and that of a traditional structured group. In basic SGRP the output is an individual's opinion (potentially) revised on the basis of seeing several other opinions, but for traditional structured groups the output is the aggregate of the (potentially) revised opinions of all the group members23. So, as we have previously noted, the SGRP arrangement is more akin to JAS than Delphi: a participant is offered some expert advice and determines whether or not to revise his or her original view. Since our basic motivation for developing the SGRP is to assist research into factors affecting opinion change, this 'limitation' does not trouble us. Further, as we have

23 In which case you have just one data point per group. An alternative would be to take each individual group member's revised opinion as the unit of analysis, but unlike the SGRP output these observations are not independent of each other, so doing this does not help much to increase analytic power. 
discussed above, JASs are themselves important judgment-aiding technologies and the subject of a significant research literature (see Bonaccio \& Dalal, 2006, for a review).

\section{Can SEE participants also take part in the main study?}

Participants in the SEE can be used in a number of ways. First, they could continue to be used in a traditional study, for example, receiving feedback from their 'real' nominal group/s in a second round (and so on). This might be a sensible ploy if real experts have been recruited, and the results would still be of use in establishing their particular views on the central topic of the survey. Second, they could, after the first round, be informed that there is in fact no second round (but see the point below about deception), with their involvement ending at that point. Third, they could continue to be used in the study proper, but rather than receiving feedback from some pre-determined nominal group (a subset of the recruited panellists), they would then receive feedback from their own SEE database (or indeed, data from previous entries in this database) according to the experimental intent and design of the study. The latter approach seems the most efficient use of recruited panellists, although there might be logistical difficulties in rapidly entering their preliminary data into the SEE database and identifying apt feedback without the length of inter-round gap becoming excessive. This approach might be best for studies involving Random Selection, and least good for those involving Informed Selection (the latter needing the greatest amount of data treatment).

\section{Can there be more than two rounds in the SGRP study?}

The SGRP as described here relates to two-round protocols only, which are prevalent in practice. However, it is perfectly possible that the approach could be extended for use in studies with three or more rounds. There are a number of ways in which this could be accomplished, including: use of confederates; constructing plausible revised answers; selecting appropriate revised answers from previous studies; and having the same participants respond over the multiple SGRP rounds while maintaining the integrity of the virtual groups they see at each round (cf re-using SEE participants as discussed in the answer to the previous question): the precise method used would depend on the research questions being posed and the characteristics of the experts and judgment task. Such studies might reveal important additional influences on VOC, such as how people with particular personal characteristics respond to certain types of feedback, however, adding further rounds increases complexity and potentially reduces some of the benefits of SGRP relative to traditional structuredgroup methods with real groups, as we argued in the answer to the first question above. We therefore submit 
that extending the SGRP to additional rounds is superfluous at this time, when there is still so much to learn about changes by individuals to initial feedback from nominal others.

\section{How many participants do you need in the SEE, and of what sort?}

If external validity is a concern, then it is important to ensure that the dataset used in the main experiment truly includes the full and relevant range of responses - which might not be the case if the SEE were conducted with an especially small sample, or with a sample that was in some way unusual or biased (e.g. experts from a single country or industry where the issue is of relevance to multiple countries or industries). As an example, consider making a forecast of a geopolitical event. You might conduct the SEE exercise with, say, 30 participants, asking for rationales regarding why that event might occur, and why it might not occur. If a small range of rationales were uncovered that were frequently mentioned, then you might feel confident that you had acquired most of the issues via the SEE. However, if there were a large range of rationales produced, few of which were repeated, then this might cause some concern that the elicitation exercise hadn't fully captured the real-life richness of factors on which people would likely judge that particular problem. In such a case we would recommend further sampling of subjects in a rolling SEE until 'saturation' is reached - a concept used in qualitative social science research where data is gathered (e.g. in interviews or focus groups) until no further significant themes are produced. For an extensive discussion of the saturation concept and methods, see Saunders et al. (2018). Unfortunately, since each judgment or reasoning task is different, devising a universal rule for calculating SEE sample size seems to us infeasible. The critical point about sample size for the SEE is how many different valid responses (or response types) there are in the population. If only one response or type of response is possible (or few, as in the case of n-alternative forced choice tasks) then you may not need to do a SEE at all (you may need a SEE to elicit rationales for these responses, though). However, in most cases there will be a finite number of possible valid responses, but that number will not be known a priori - we contend that saturation is the only way to find out 24 .

\footnotetext{
24 Incidentally, it is worth noting that a small number of different responses can be combined into a large number of unique groups (i.e. where each different response is included just once per group). For example, although 10 different responses only gives 210 unique groups of 4 experts, and 45 groups of 8, increase the number of responses to 30 and the numbers of unique groups is 27,405 and 5,852,925 for 4 and 8 member groups respectively. See

https://stattrek.com/probability/combinations-permutations.aspx
} 


\section{Are we not deceiving the participants in both phases of the SGRP?}

Ideally, panellists recruited for a SEE should be informed that they are part of a Delphi group in the same way they would be in a 'real' Delphi exercise to ensure a similar level of motivation and expectation that their responses will be read and used by others (as they will be). If SEE participants are not to receive feedback and have subsequent responses collected, then it might well suffice to inform them that they are simply providing information for others rather than being in a nominal group per se: whether this reduces the quality of their responses is an empirical question, though it is difficult to see why this should have a devastating effect on what they produce. Even if the experimenter wished to take no chances and initially suggest that SEE participants would receive feedback in a Delphi group, and then subsequently tell them they would not, full debriefing at the end of the exercise would likely be a sufficient ethical response, with mild deception not generally considered problematic in psychological studies. This also goes for participants in the main SGRP study (Phase 2), although it is likely that their instructions concerning receiving information from nominal others can be framed in an accurate manner in all but the most contrived scenarios (i.e. where feedback is largely created de novo rather than being based on the actual responses of SEE participants).

\section{How relevant is the SGRP for understanding opinion change in groups beyond Delphi and JAS?}

The mapping of the second phase of the SGRP onto a prototypic 2-round Delphi or JAS protocol should be clear, and we have indicated how additional rounds of a Delphi could potentially be accommodated, however, the relevance to understanding opinion change in other structured-group techniques, or groups more generally may not be so apparent. As we have indicated above, the main way in which Delphi and JAS deviate from the other methods we have discussed is that, for the most part, they more strictly control the nature of the interaction between group members. Two ways in which 'direct' interactions might differ from the controlled feedback of Delphi include: the formation of coalitions between group members; and the creation of different roles and/or hierarchies (e.g. leaders and followers). As we discussed in the Introduction, these are both potential sources of bias, and thus the reason for being excluded from Delphi. However, we do not know how biasing these things actually are in practice, nor if their effect is always negative. For example, perhaps in some circumstances emergence of a strong leader can help break a deadlock and facilitate better outcomes? Or a minority voice might become stronger on finding an ally and thus overturn a mistaken majority? We believe that the SGRP could help answer these questions by enabling the effects of specific features of interactions 
between group members to be investigated. For instance, coalitions of similar opinions can be explicitly created and compared against no explicit coalitions, or no possible coalitions (i.e. a set of disparate viewpoints). Similarly, roles can be allocated to both real and simulated participants and compared with conditions with different role allocations or no role allocations. Although such manipulations may also be possible with real interacting groups by, for instance, using confederates, or preselecting group members on the basis of particular traits or opinions, it is likely to be much easier and more efficient (i.e. require fewer participants) with the SGRP.

Slowing down or reducing the amount or quality of information exchange as a result of restricting the interaction in structured groups, might also have negative effects on performance - these can also be investigated in the SGRP. For instance, written presentation of reasons could be compared to spoken, graphical or various combinations thereof, while quantity and quality can also readily be manipulated, as we have already discussed above. Naturalistic exchanges such as conversations between experts would be harder to simulate, but not impossible. For instance, interchanges between pairs of experts could be captured in the SEE and then presented during the main study, or participants could be allowed to select from a menu of question topics with pre-scripted answers also taken from a SEE. Performance could then be compared to that of a few real groups, thereby permitting the sources of any detriment in the simulated versions to be readily identified by virtue of the small remaining differences between the simulated and naturalistic conditions. In this way the SGRP can also shed light on processes of opinion change in directly interacting groups.

Finally, PMs have the most restricted information exchange of any of the methods discussed here: typically participants can only see the current market price - and offered buy and sell prices - and indicate their opinion of those prices (and hence the likely outcome of the market) by the offers they make, and the deals they strike.25 PMs are therefore like a Delphi where aggregate information is fed back (e.g. mean and range of prices) and nothing else. Since PMs usually have hundreds or thousands of participants it would be difficult for participants to process information from individual traders (even if there was an incentive for useful information to be tendered), but it is possible to imagine a PM where enriched feedback is given, for

25 Exactly how a market works depends on the particular platform, but this is the case on one of the largest, PredictIt. PredictIt also allows for comments, but in our experience the value of the discourse for accurate pricing is questionable (e.g. largely consisting of partisan sniping in election markets): as we have commented earlier, PMs are competitive rather than cooperative so disinformation is more likely than information. 
example, the variation in prices, or a summary of rationales for particular 'unusual' prices. The SGRP could be used to investigate whether such innovations improve the markets. While PMs are fairly easy to recruit to in sufficient numbers to operate reliably - being 'fun' and mostly without the requirement for participants to have particular expertise - they lack the control needed to get to the nitty gritty of what works best and why. The SGRP permits the manipulation of important aspects of traders (e.g. expertise, confidence, competitiveness), markets (e.g. number of options, predictability, consensus), and platforms (e.g. amount, type and rapidity of feedback) in experimental designs that have sufficient power to answer research questions with hundreds rather than thousands of participants in the main study. But do we not need to run thousands in the SEE in order to get realistic data for feedback in the main study? Not necessarily. While one option would certainly be to use data from real PMs - either previously run for analogous markets or one specifically run for the SEE - an alternative could be to build models of markets of various kinds then use these models to generate simulated trades etcetera. The models could be validated using historic data and then used to produce plausible data for feedback in SGRP main studies.

\section{Summary and Conclusions}

The main aim of this paper has been to propose a new research approach to the study of opinion change in Delphi and other structured groups, introducing our Simulated Group Response Paradigm (SGRP). We developed this approach in order to help researchers answer the call of Rowe and Wright (2011) to conduct a greater number of more rigorous studies into the working mechanisms of Delphi so as to inform the application of the method and thus maximise its potential for improving human judgment and decision making: we have also argued that the SGRP has relevance to understanding and improving structured-group techniques more generally. To this end we identified a variety of factors that should be the source of future research (see Figure 1), the study of which will be enabled by our paradigm - the traditional approach with 'real' groups suffering as it does from a lack of experimental control and insufficient power for suitable analysis, unless a generally unfeasible number of participants are recruited.

We believe that research into opinion formation and change in online groups is particularly timely due to the huge increase in group-working over the internet resulting from the novel coronavirus pandemic: such working is likely to remain more common than beforehand after the pandemic recedes, due to realised gains in productivity and an associated reduction of carbon emissions. Even prior to COVID-19, recent years have 
seen a burgeoning of online meetings for commercial, government and academic purposes, as well as the emergence of numerous public fora and communities such as Quora, Reddit, Mumsnet, TripAdvisor and Change My View, to name but a few. We submit that research using the SGRP can help investigate how such online information exchanges and opinion makers can lead to improved decision making in organizations and wider society (e.g. for promoting desired environmental and health behaviours, or increasing public participation in government) rather than perpetuating - or even amplifying - bias, prejudice and misinformation.

One additional possibility that we find exciting is the prospect of researchers throughout the world using SEEs for a range of problems (judgmental, decision making and problem solving) and then making their databases accessible more widely. This approach could significantly aid the Delphi and nominal group research community by shortening the research process (as researchers would not all have to conduct their own SEE), aiding consistency, and potentially leading to a quantum leap in the number of rigorously conducted, well-analysed studies. Furthermore, such studies, we hope, would likely be aimed at answering more sophisticated questions than "does technique x 'work'?", notably addressing the relative utility of different variations and compositions of structured-group procedures and their capacity to lead to virtuous opinion change (VOC) in individuals. Results from such research could then be used inform the conduct of real-world structured-group instantiations seeking specific answers to policy issues etcetera, thereby increasing the likelihood of producing high quality answers to important questions.

To conclude, we commend this novel paradigm to the research community and invite others to adopt, revise, and extend it as they see fit.

\section{Acknowledgements:}

This research is based on work supported in part by the Office of the Director of National Intelligence (ODNI), Intelligence Advanced Research Projects Activity (IARPA), under Contract [2017-16122000003].

The views and conclusions contained herein are those of the authors and should not be interpreted as necessarily representing the official policies, either expressed or implied, of ODNI, IARPA, or the U.S. Government. The U.S. Government is authorized to reproduce and distribute reprints for governmental purposes notwithstanding any copyright annotation therein. 


\section{References:}

Alvarado-Valencia, J., Barrero, L.H., Onkal, D. \& Dennerlein, J.T. (2017). Expertise, credibility of systems forecasts and integration of methods in judgmental demand forecasting. International Journal of Forecasting, 33(1), 298-313.

Arnott, D. (2006). Cognitive biases and decision support systems development: A design science approach. Information Systems Journal, 16(1), 55-78.

Asch, S.E. (1951). Effects of group pressure on the modification and distortion of judgments. In H. Guetzkow (Ed.), Groups, Leadership and Men (pp. 177-190). Pittsburgh, PA: Carnegie Press.

Birko, S., Dove, E.S. \& Ozdemir, V. (2015). Evaluation of nine consensus indices in Delphi foresight research and their dependency on Delphi survey characteristics: A simulation study and debate on Delphi design and interpretation. PLoS One, 10, p. e0135162.

Blascovich, J., Mendes, W.B., Hunter, S.B., \& Salomon, K. (1999). Social "facilitation" as challenge and threat. Journal of Personality and Social Psychology, 77(1), 68-77.

Boje, D.M., \& Murnighan, J.K. (1982). Group confidence pressures in iterative decisions. Management Science, 28(10), 1187-1196.

Bolger F. (2018). The selection of experts for (probabilistic) expert knowledge elicitation. In L. Dias, A. Morton \& J. Quigley (Eds.), Elicitation. International Series in Operations Research \& Management Science, vol 261. (pp. 393-443). Cham, Switzerland: Springer.

Bolger, F. \& Rowe, G. (2015a). The aggregation of expert judgment: Do good things come to those who weight? Risk Analysis, 35(1), 5-11.

Bolger, F. \& Rowe, G. (2015b). There is data, and then there is data: Only experimental evidence will determine the utility of differential weighting of expert judgment. Risk Analysis, 35 (1), 21-26.

Bolger, F. \& Wright, G. (2011). Improving the Delphi process: Lessons from social psychological research. Technological Forecasting and Social Change, 78, 1500-1513.

Bolger, F. \& Wright, G. (2017). Use of expert knowledge to anticipate the future: Issues, analysis and directions. International Journal of Forecasting, 33(1), 230-243. 
Bonaccio, S. \& Dalal, R.S. (2006). Advice taking and decision-making: An integrative literature review, and implications for the organizational sciences. Organizational Behavior and Human Decision Processes, $101,27-151$

Brabham, D.C. (2008). Crowdsourcing as a model for problem solving: An introduction and cases. Convergence: The International Journal of Research into New Media Technologies, 14(1), 75-90.

Brockhoff, K. (1975). The performance of forecasting groups in computer dialogue and face-to-face discussion. In H. Linstone \& M. Turoff (Eds.), The Delphi Method: Technique and Applications. London: Addison-Wesley.

Brunswik, E. (1955). Representative design and probabilistic theory in a functional psychology. Psychological Review, 62(3), 193.

Buck, R., Losow, J.I., Murphy, M.M., \& Costanzo, P. (1992). Social facilitation and inhibition of emotional expression and communication. Journal of Personality and Social Psychology, 63(6), 962.

Burgman, M.A., McBride, M., Ashton R., Speirs-Bridge, A., Flander, L., et al. (2011). Expert status and performance. PLoS ONE, 6(7), e22998.

Chidambaram, L., \& Tung, L.L. (2005). Is out of sight, out of mind? An empirical study of social loafing in technology-supported groups. Information Systems Research, 16(2), 149-168.

Dalkey, N.C. (1975). Toward a theory of group estimation. In: H.A. Linstone, M. Turoff (Eds.), The Delphi Method: Techniques and Applications, (pp. 236-261). Reading, MA: Addison-Wesley.

Dalkey, N. \& Helmer, O. (1963). An experimental application of the Delphi Method to the use of experts. Management Science, 9(3), 458-467.

Delbecq, A.L. \& Van de Ven, A.H. (1971). A group process model for problem identification and program planning. Journal of Applied Behavioral Science, 7, 466-91.

Dhami, M.K., Belton, I.K. \& Careless, K.E. (2016). Critical review of analytic techniques. In 2016 European Intelligence and Security Informatics Conference (EISIC) (pp. 152-155). IEEE.

Dias, L.C., Morton, A. \& Quigley, J. (2018). Elicitation: The Science and Art of Structuring Judgment. Cham, Switzerland: Springer International Publishing. 
Dodoiu, G., Leenders, R.T. \& van Dijk, H. (2016). A meta-analysis of whether groups make more risky or more cautious decisions than individuals. Academy of Management Proceedings, 2016(1), 16461.

The Economist (2016). Who said Brexit was a surprise? The Economist, 24 June. ISSN 0013-0613.

Edwards, W., Lindman, H., \& Phillips, L.D. (1965). Emerging technologies for making decisions. In F. Barron et al. (Eds.), New Directions in Psychology II (pp. 261-325). New York, Holt, Rinehart \& Winston.

Efrati, O. (2019). The rise of crowdsourcing: It gets the people going. International Business Times, June 28. https://www.ibtimes.com/rise-crowdsourcing-it-gets-people-going-2803310

European Food Safety Authority (EFSA) (2014). Guidance on expert knowledge elicitation in food and feed safety risk assessment. EFSA Journal, 12(6), 3734.

Graefe, A., \& Armstrong, J.S. (2011). Comparing face-to-face meetings, nominal groups, Delphi and prediction markets on an estimation task. International Journal of Forecasting, 27(1), 183-95.

Gelman, A. \& Rothschild, D. (2016). Something's odd about the political betting markets. Slate, 12 July. ISSN 1091-2339.

Gibbons, A.M., Sniezek, J.A. \& Dalal, R.S. (2003). Antecedents and consequences of unsolicited versus explicitly solicited advice. In D. Budescu (Chair), Symposium in Honor of Janet Sniezek, annual meeting of the Society for Judgment and Decision Making, Vancouver, BC. 2003 (November).

Gordon, T.J. \& Pease, A. (2006). RT Delphi: An efficient, "round-less", almost real time Delphi method. Technological Forecasting and Social Change, 73(4), 321-333.

Hackman, J.R. \& Katz, N. (2010). Group behavior and performance. In S. T. Fiske, D. T. Gilbert \& G. Lindzey (Eds.), Handbook of Social Psychology, Vol. 2, 5th edition (pp. 1208-1251). Hoboken, NJ: John Wiley \& Sons.

Hackman, J. \& Morris, C. (1975). Group tasks, group interaction processes, and group performance effectiveness: A review and proposed integration. In L. Berkowitz (Ed.), Advances in experimental social psychology, Vol. 8 (pp. 45-99). New York: Academic Press.

Hanea, A., McBride, M., Burgman, M., Wintle, B., Fidler, F., Flander, L., Twardy, C.R., Mascaro, S. \& Manning, B. (2016). InvestigateDiscussEstimateAggregate for structured expert judgement. International 
Journal of Forecasting, 33(1), 267-9.

Harvey, N., \& Fischer, I. (1997). Taking advice: Accepting help, improving judgment, and sharing responsibility. Organizational Behavior and Human Decision Processes, 70(2), 117-133.

Hasson, F. \& Keeney, S. (2011). Enhancing rigour in the Delphi technique research. Technological Forecasting and Social Change, 78(9), 1695-1704.

Hastie, R. \& Kameda, T. (2005). The robust beauty of majority rules in group decisions. Psychological Review, 112(2), 494-508.

Haynes, C.A., \& Shelton, K. (2018). Delphi method in a digital age: Practical considerations for online Delphi studies. In Handbook of Research on Innovative Techniques, Trends, and Analysis for Optimized Research Methods (pp. 132-151). IGI Global.

Johnson, D.D. \& Fowler, J.H. (2011). The evolution of overconfidence. Nature, 477(7364), 317-20.

Kahneman, D., Slovic, P., \& Tversky, A. (1982). Judgment Under Uncertainty: Heuristics and Biases. Cambridge: Cambridge University Press.

Kuhn, T. (1962). The Structure of Scientific Revolutions. Chicago: Chicago University Press.

Levingston, I. (2016). Why political polls and betting odds disagree with each other so much, CNBC, 28 July.

Lichtenstein, S., Fischhoff, B., \& Phillips, L.D. (1982). Calibration of probabilities: The state of the art to 1980. In D. Kahneman, P. Slovic, \& A. Tversky (Eds.), Judgment Under Uncertainty: Heuristics and Biases (pp. 306-334). Cambridge: Cambridge University Press.

Linstone, H.A. \& Turoff, M. (1975). The Delphi Method: Techniques and Applications. London: Addison-Wesley.

Markus, H. (1978). The effect of mere presence on social facilitation: An unobtrusive test. Journal of Experimental Social Psychology, 14, 389-397.

McGrath, J.E. (1984). Groups: Interaction and Performance. Englewood Cliffs, NJ: Prentice Hall.

Meijering, J.V., Kampen J.K. \&Tobi H. (2013). Quantifying the development of agreement among experts in Delphi studies. Technological Forecasting and Social Change, 80, 1607-1614. 
Moscovici, S. (1985). Social influence and conformity. In G. Lindzey \& E. Aronson (Eds.), Handbook of Social Psychology (3rd ed., pp. 347-412). New York, NY: Random House.

Parenté, F.J. \& Anderson-Parenté, J.K. (1987). Delphi inquiry systems. In: G. Wright, P. Ayton (Eds.), Judgmental Forecasting (pp. 129-156). Chichester, Wiley.

Parenté, R.J., Hiöb, T.N., Silver, R.A., Jenkins, C., Poe, M.P., \& Mullins, R.J. (2005). The Delphi method, impeachment and terrorism: Accuracies of short-range forecasts for volatile world events. Technological Forecasting and Social Change, 72(4), 401-411.

Phillips L.D. (1999). Group elicitation of probability distributions: Are many heads better than one? In: J. Shanteau, B.A., Mellers \& D.A. Schum (Eds), Decision Science and Technology. Springer, Boston, MA.

Price, P.C., \& Stone, E.R. (2004). Intuitive evaluation of likelihood judgment producers: Evidence for a confidence heuristic. Journal of Behavioral Decision Making, 17(1), 39-57.

Rothstein, H., Sutton, A.J. \& Borenstein, M. (2005). Publication bias in meta-analysis: Prevention, assessment and adjustments. Wiley. Chichester, England.

Rowe, G. \& Wright, G. (1996). The impact of task characteristics on the performance of structured group forecasting techniques. International Journal of Forecasting, 12, 73-89.

Rowe, G. \& Wright, G. (1999). The Delphi technique as a forecasting tool: Issues and analysis, International Journal of Forecasting, 15, 353-375.

Rowe, G. \& Wright, G. (2011). The Delphi Technique: Past, present, and future prospects - introduction to the Special Issue. Technological Forecasting and Social Change, 78(9), 1487-1490.

Rowe, G., Wright, G. \& Bolger, F. (1991). The Delphi technique: A re-evaluation of research and theory, Technological Forecasting and Social Change, 39(3), 235-251.

Rowe, G., Wright, G., \& McColl, A. (2005). Judgment change during Delphi-like procedures: The role of majority influence, expertise, and confidence. Technological Forecasting and Social Change, 72(4), 377-399.

Saunders, B., Sim, J., Kingstone, T., Baker, S., Waterfield, J., Bartlam, B., Burroughs, H. \& Jinks, C. (2018). Saturation in qualitative research: Exploring its conceptualization and operationalization. Quality and Quantity, 52(4), 1893-1907. 
Sah, S., Moore, D.A. \& MacCoun, R.J. (2013). Cheap talk and credibility: The consequences of confidence and accuracy on advisor credibility and persuasiveness. Organizational Behavior and Human Decision Processes, 121(2), 246-255.

Sniezek, J.A. \& Buckley, T. (1995). Cueing and cognitive conflict in judge-advisor decision making. Organizational Behavior and Human Decision Processes, 62, 159-174.

Sniezek, J.A., Schrah, G.E., \& Dalal, R.S. (2004). Improving judgment with prepaid expert advice. Journal of Behavioral Decision Making, 17, 173-190.

Straus, S.G. (1999). Testing a typology of tasks: An empirical validation of McGrath's (1984) group task circumplex. Small Group Research, 30(2), 166-187.

Stasser, G. \& Vaughan, S.I. (2013). Models of participation during face-to-face unstructured discussion. In E.H. Witte \& J.H. Davis (Eds.), Understanding Group Behavior: Consensual Action by Small Groups, Vol. 1 (pp 165-192). New York: Psychology Press.

Steiner, I.D. (1972). Group Process and Productivity. New York: Academic Press.

Stoner, J.A. (1968). Risky and cautious shifts in group decisions: The influence of widely held values. Journal of Experimental Social Psychology, 4(4), 442-459.

Surowiecki, J. (2005). The Wisdom of Crowds. New York: Anchor Books.

Swaab, R. I., Phillips, K. W., \& Schaerer, M. (2016). Secret conversation opportunities facilitate minority influence in virtual groups: The influence on majority power, information processing, and decision quality. Organizational Behavior and Human Decision Processes, 133, 17-32.

Turoff, M. (1970). The design of a policy Delphi. Technological Forecasting and Social Change, 2(2), $149-171$

von der Gracht, H. (2012). Consensus measurement in Delphi studies: Review and implications for future quality assurance. Technological Forecasting and Social Change, 79(8), 1525-1536.

Weber, B. \& Hertel, G. (2007). Motivation gains of inferior group members: A meta-analytical review. Journal of Personality and Social Psychology, 93(6), 973-993.

Yaniv, I. \& Kleinberger, E. (2000). Advice taking in decision making: Egocentric discounting and 
reputation formation. Organizational Behavior and Human Decision Processes, 83, 260-281.

Zajonc, R. B. (1965). Social facilitation. Science, 149, 269-274.

Zajonc, R. B. (1980). Feeling and thinking: Preferences need no inferences. American Psychologist, 35(2), 151. 


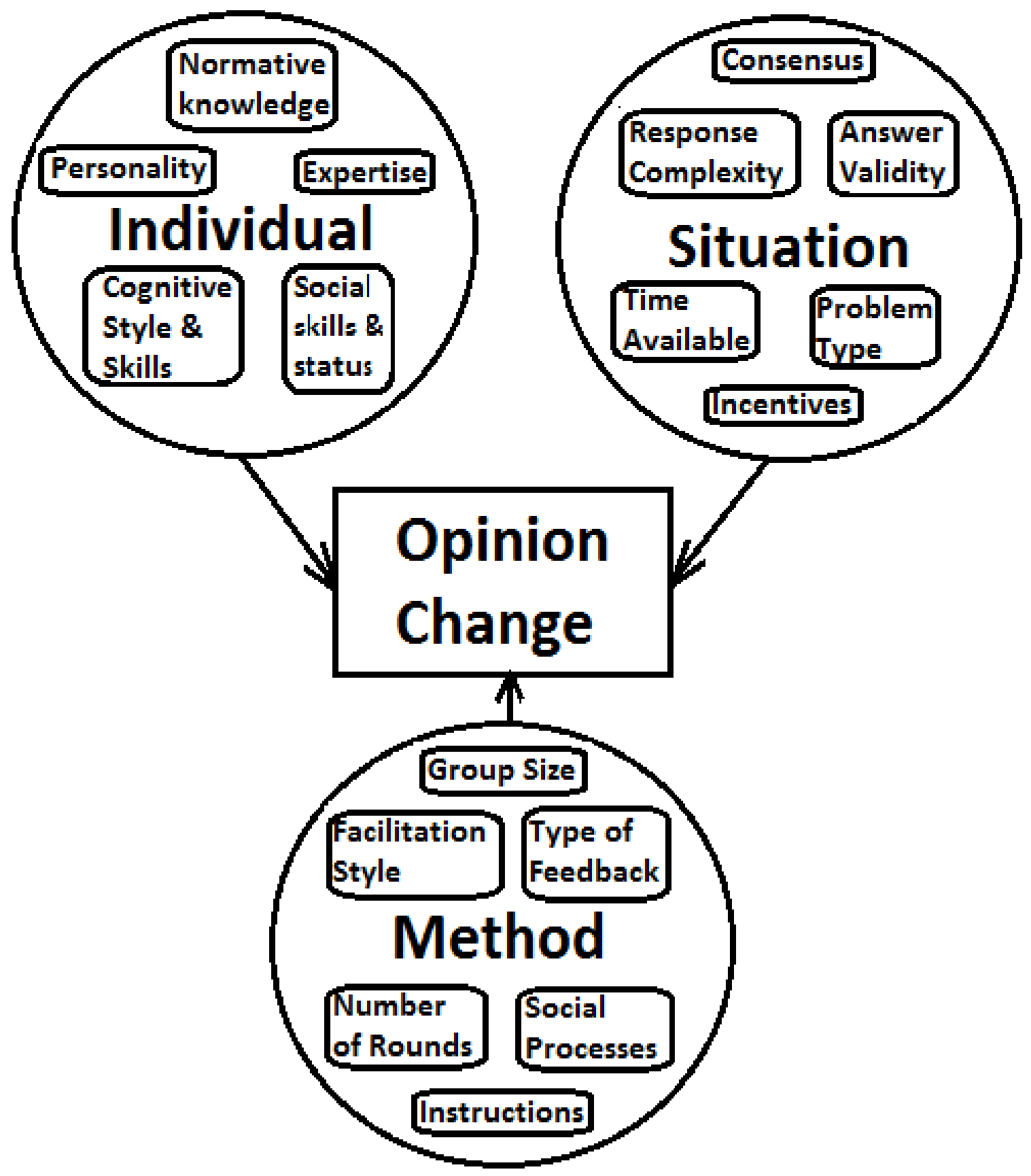

Figure 1: Some factors potentially influencing opinion change of individuals in structured-group techniques. 


\begin{tabular}{|c|c|c|c|}
\hline $\begin{array}{l}\text { PHASE 1: Stimulus } \\
\text { Creation/Collection }\end{array}$ & $\begin{array}{c}\text { The SEE } \\
\text { [optional steps in brackets] }\end{array}$ & $\begin{array}{l}\text { Stimulus Selection } \\
\text { during curation }\end{array}$ & $\begin{array}{c}\text { PHASE 2: } \\
\text { Experimentation }\end{array}$ \\
\hline
\end{tabular}

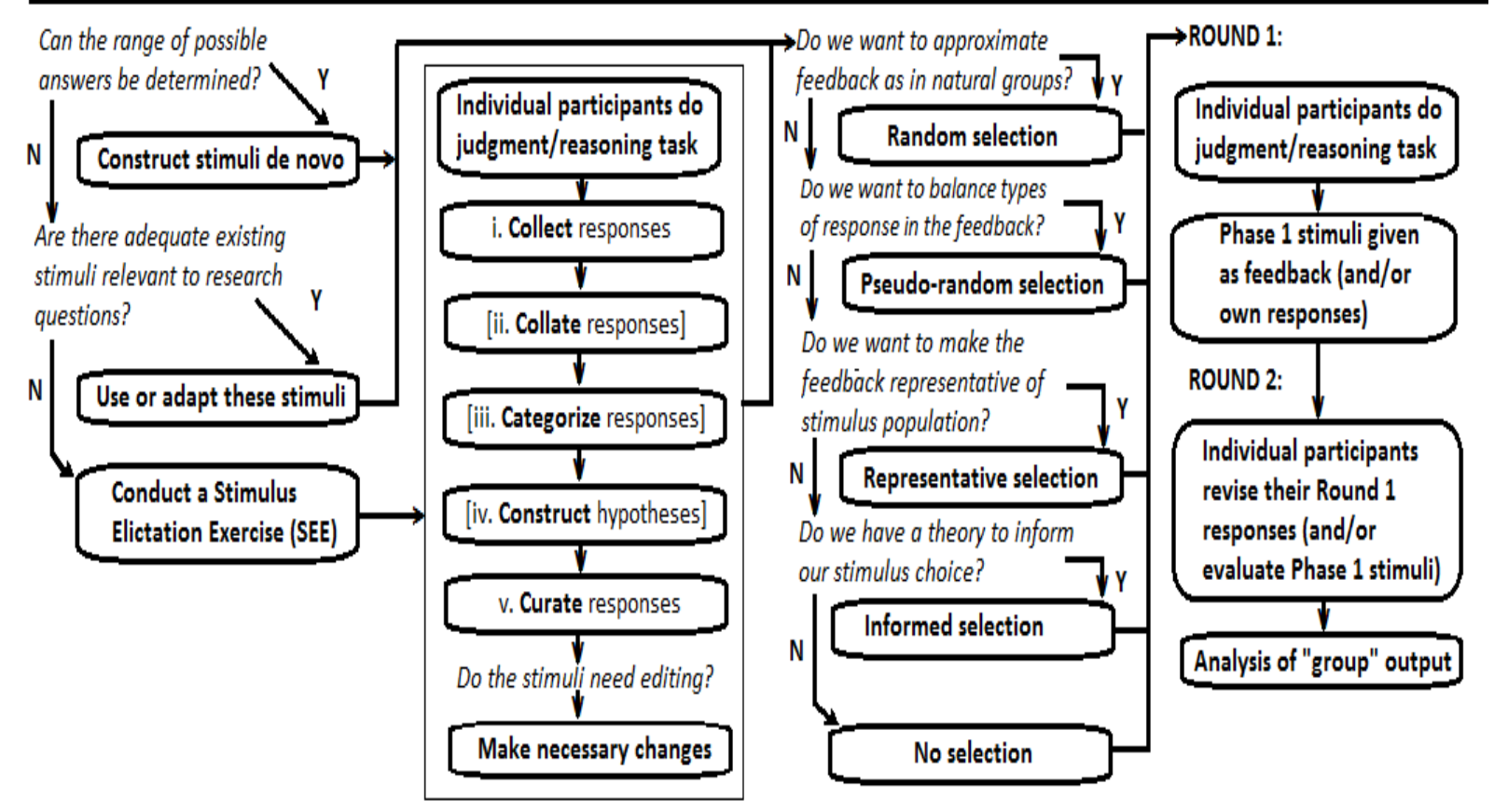

Figure 2: The Simulated Group Response Paradigm (SGRP) 